\title{
Calbindin-D9k in Hypoxia-Induced Dabetes Mellitus like Model
}

Changhwan Ahn, Kipoong Kim, Song Ai Kang, Tran Dinh Nam and Eui-Bae Jeung

Laboratory of Veterinary Biochemistry and Molecular Biology, Chungbuk National University, Cheongju, Chungbuk, 28644, Republic of Korea

\section{Introduction}

Introduction: It has been proposed that cellular $\mathrm{Ca} 2+$ signals activate hormone secretion. In pancreatic $\beta$ cells, which produce insulin, $\mathrm{Ca}+$ signals have been known to contribute to insulin secretion. Prior to this study, we confirmed that Calbindin-D9k (CaBP-9k) was responsible for regulation of the distribution of free calcium in the cytoplasm. We also confirmed that insulin-secreting $\beta$ cells express $\mathrm{CaBP}-9 \mathrm{k}$, and assumed that CaBP-9k play a role in $\beta$ cell insulin synthesis or secretion. Using CaBP-9k knock out (KO) mice, we demonstrated that ablation of CaBP-9k causes type 1 diabetes by reducing insulin secretion and increasing serum glucose.

Objective: The aim of this study was to assess interactions between glucose/insulin secretion and calcium homoeostasis in the pancreas of mice.

\section{Method}

Using CaBP-9k knock out (KO) mice, we demonstrated that ablation of CaBP-9k could causes insulin dependent diabetes by reducing insulin secretion and increasing serum glucose. In addition, to compare the role of CaBP-9k with hypoxic pathophysiological conditions, we exposed wild-type and CaBP-9k KO mice to hypoxic conditions for 10 days. Hypoxia induced endoplasmic reticulum (ER) stress, increasing both insulin signaling and insulin resistance.

\section{Result}

(A)

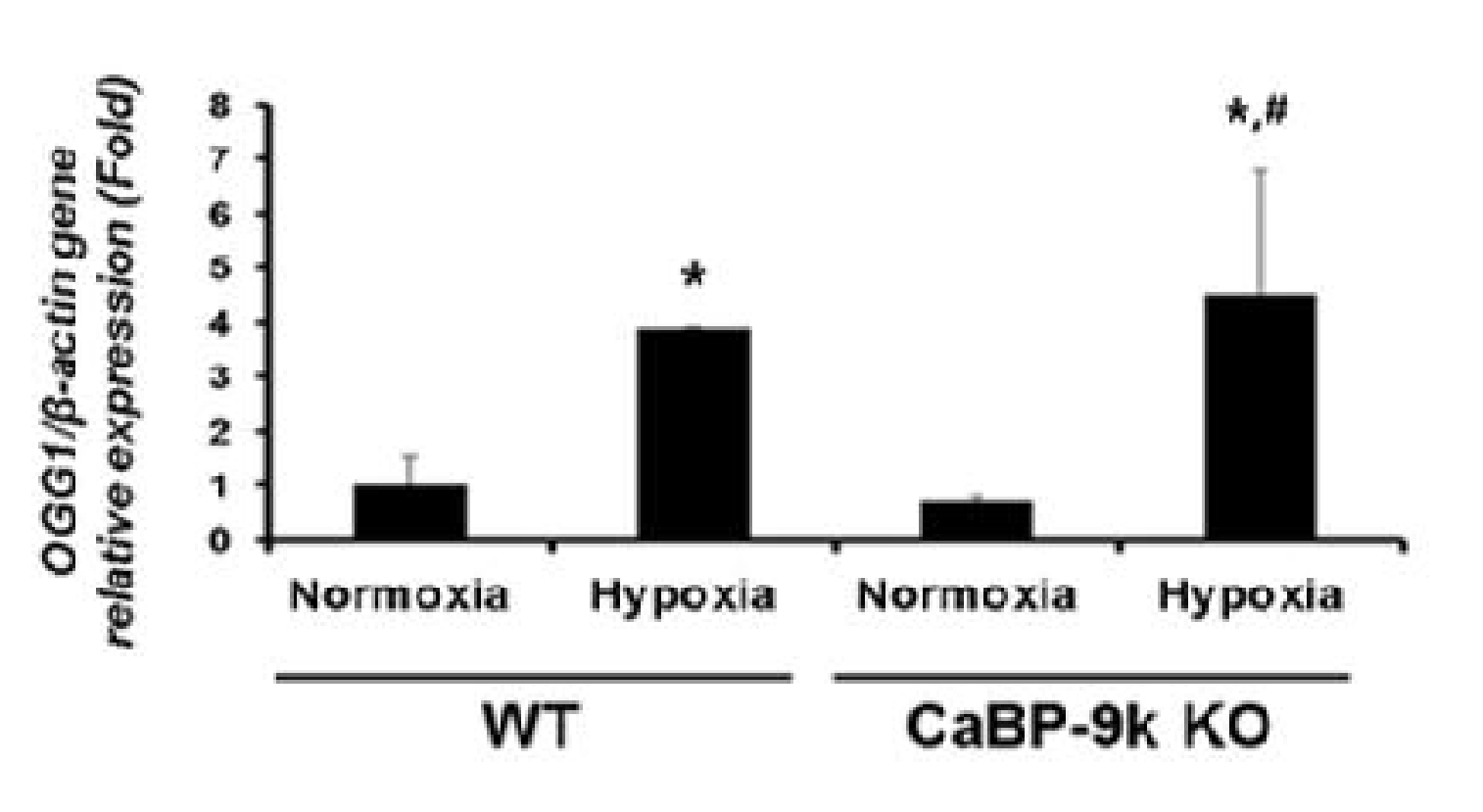

(C)

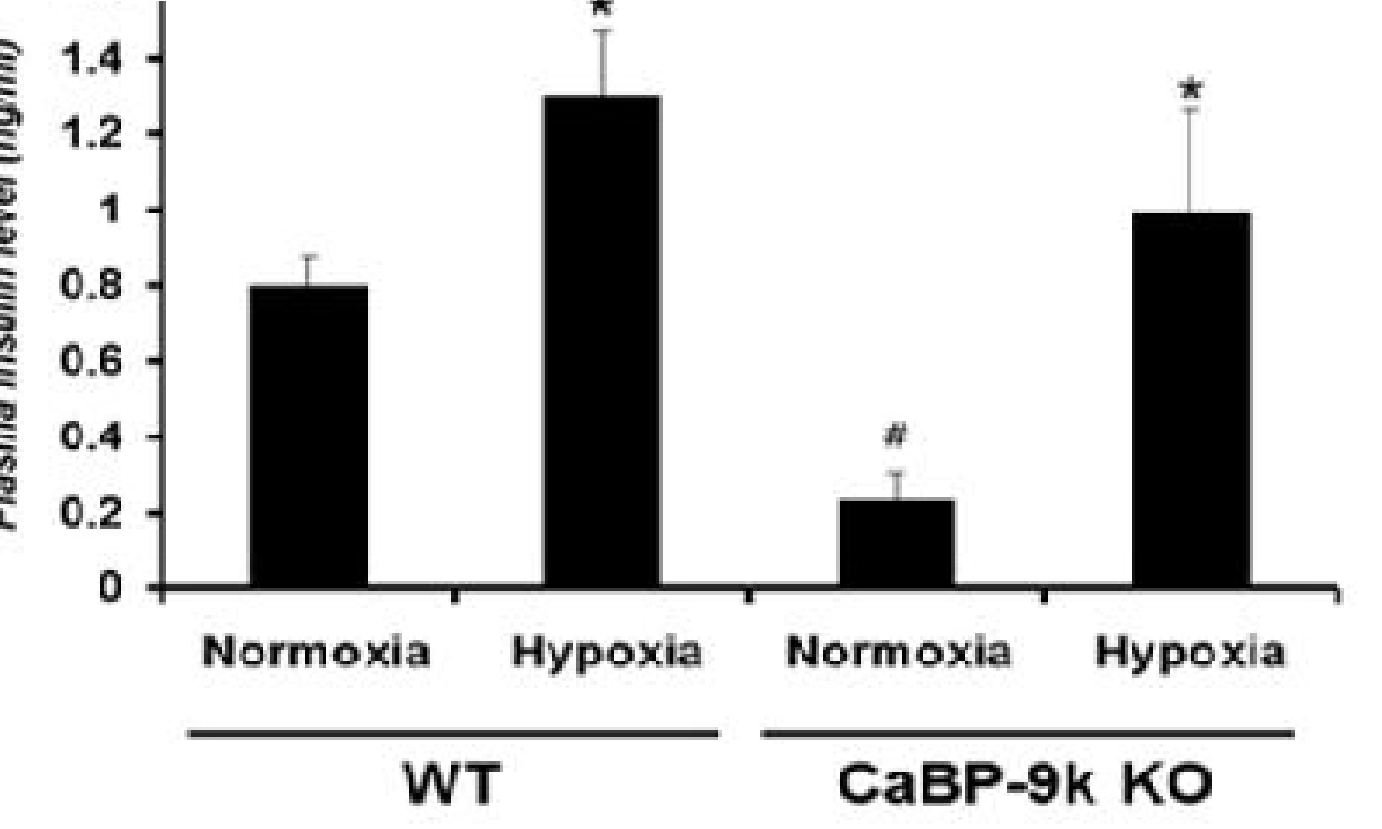

(D)

B)

$(\mathrm{E})$


(F)

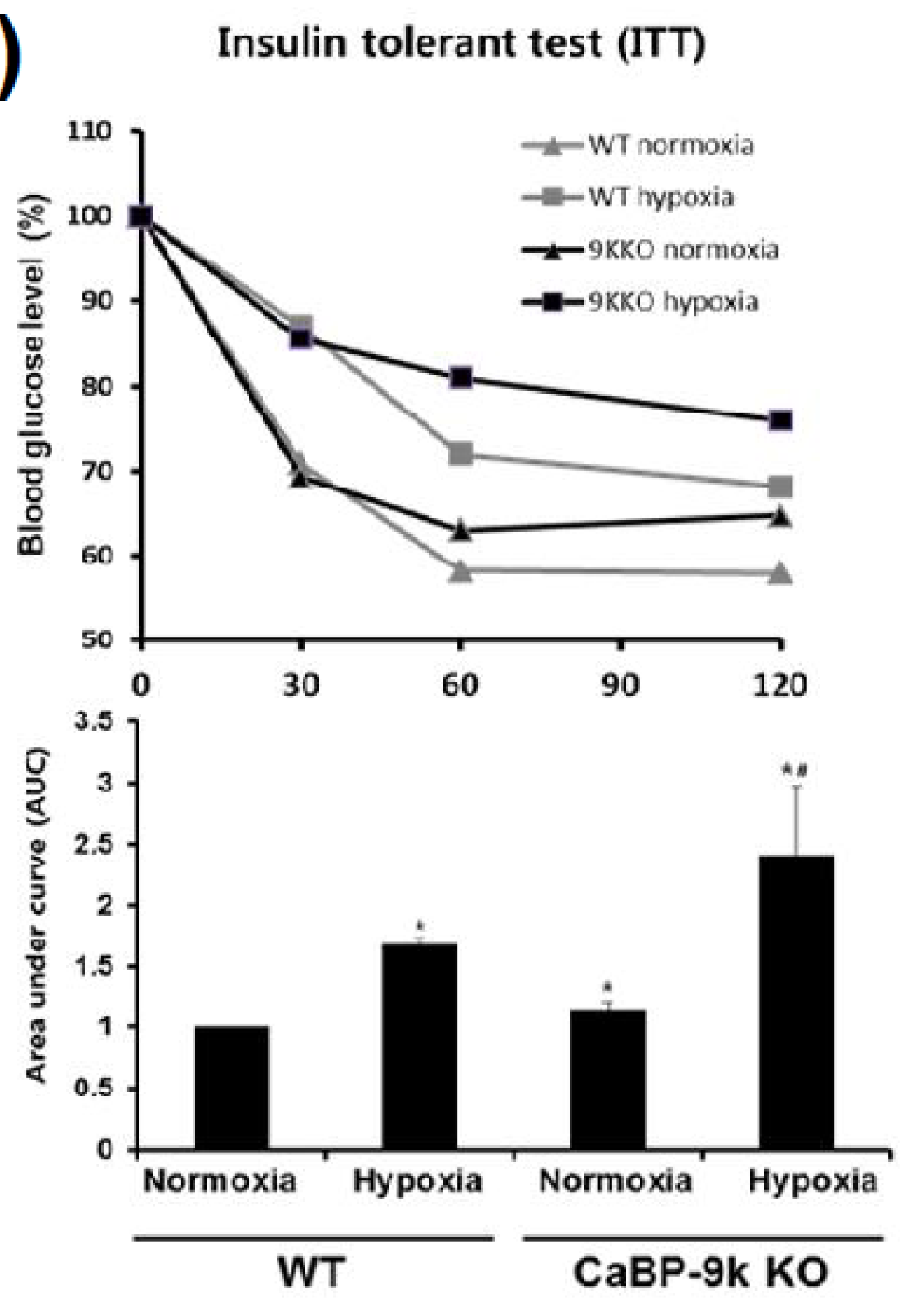

(G)


Figure 1. Regulation of glucose parameters in T2D model and CaBP-9K KO mice. (A) OGG1 expression (B) Blood glucose level. (C) Plasma insulin level. (D) Calculated insulin resistance index; HOMA-IR. (E) IPGTT and AUC (F) IPITT and AUC. (G) Immunofluorescence assay.

EP402: Diabetes

(A)

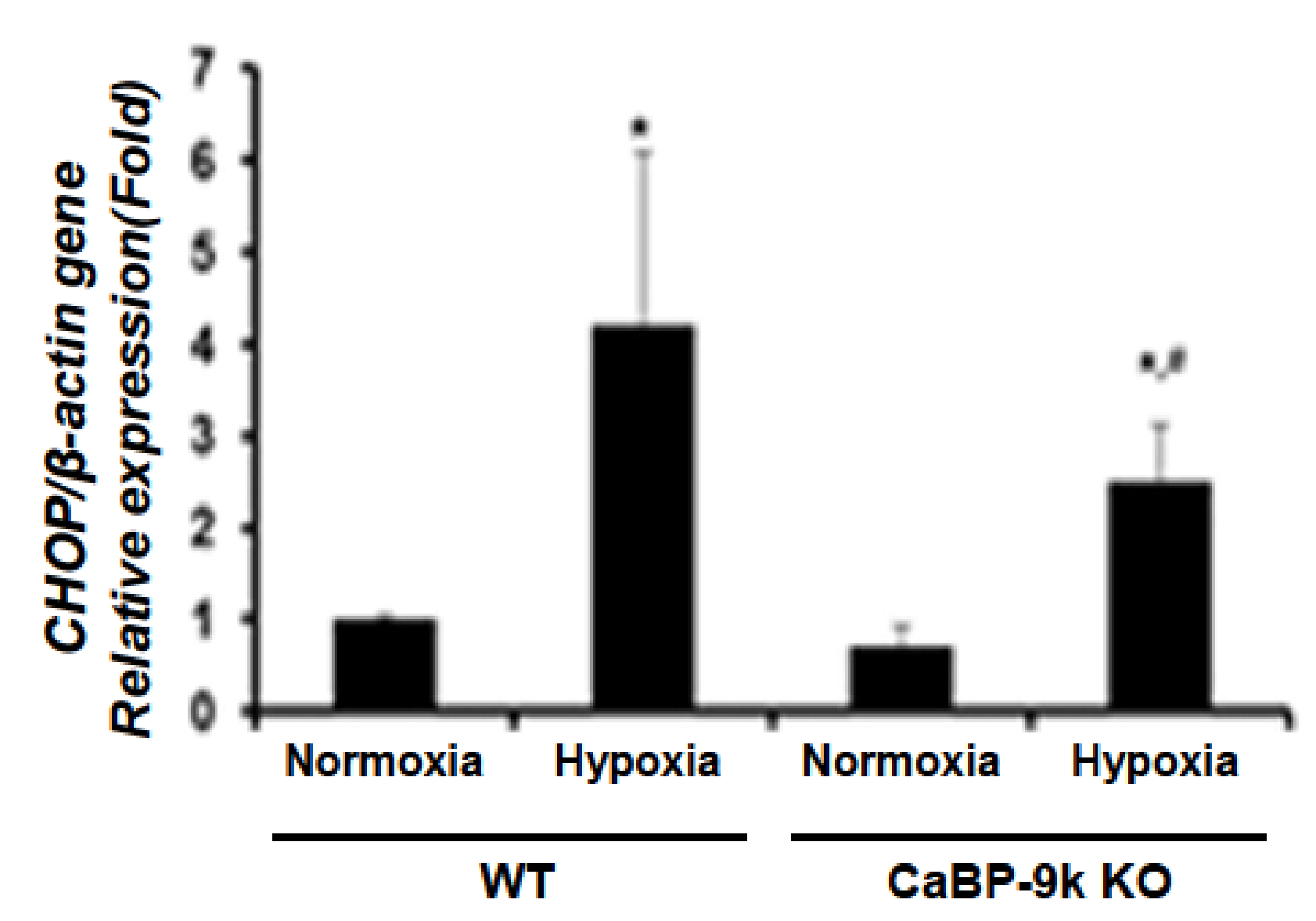

(C)



(E)


Figure 4. Expression of ER-stress maker genes in T2D model and CaBP-9K KO mice. WT and CaBP-9K KO mice were exposed to normoxic or hypoxic condition for 10 days.

\section{Conclusion}

Overall, the results of the present study demonstrated that CaBP-9k regulates synthesis of insulin and is part of the insulin-secreting calcium signaling. Therefore, impaired CaBP-9K signaling may be linked with diabetes mellitus and CaBP-9K protein is as a potential candidate for gene therapy of type 1 diabetes.

\section{Reference}

(1) Identification of Small Molecules That Protect Pancreatic $\beta$ Cells against Endoplasmic Reticulum Stress-Induced Cell Death Kim Tran, Yu Li, Hongliang Duan, Daleep Arora, Hui-Ying Lim, and Weidong Wang ACS Chemical Biology Article ASAP

(2) Calnexin Silencing in Mouse Neonatal Cardiomyocytes Induces $\mathrm{Ca}^{2+} \mathrm{Cycling}$ Defects, ER Stress, and Apoptosis Nicolas Bousette, Cynthia Abbasi, Roxana Chis, Anthony O. Gramolini Journal of Cellular Physiology 